\title{
Microcytosis is associated with low cognitive outcomes in healthy 2-year-olds in a high-resource setting
}

\author{
Elaine K. McCarthy ${ }^{1,2}$, Mairead E. Kiely ${ }^{1,2}$, Geraldine Hannon ${ }^{2}$, Caroline Ahearne ${ }^{2}$, Louise C. Kenny ${ }^{2,3}$, \\ Jonathan O'B. Hourihane ${ }^{2,4}$, Alan D. Irvine ${ }^{5,6,7}$ and Deirdre M. Murray ${ }^{2,4_{*}}$ \\ ${ }^{1}$ Cork Centre for Vitamin D and Nutrition Research, University College Cork, Cork, Republic of Ireland \\ ${ }^{2}$ The Irish Centre for Fetal and Neonatal Translational Research (INFANT), University College Cork, Cork, Republic of Ireland \\ ${ }^{3}$ Department of Obstetrics and Gynaecology, University College Cork, Cork, Republic of Ireland \\ ${ }^{4}$ Department of Paediatrics and Child Health, University College Cork, Cork, Republic of Ireland \\ ${ }^{5}$ Department of Clinical Medicine, Trinity College, Dublin, Republic of Ireland \\ ${ }^{6}$ Department of Paediatric Dermatology, Our Lady's Children's Hospital, Dublin, Republic of Ireland \\ ${ }^{7}$ National Children's Research Centre, Dublin, Republic of Ireland \\ (Submitted 15 February 2017 - Final revision received 27 June 2017 - Accepted 30 June 2017)
}

\section{Abstract}

Fe deficiency in early childhood is associated with long-term consequences for cognitive, motor and behavioural development; however explorations in healthy children from low risk, high-resource settings have been limited. We aimed to explore associations between Fe status and neurodevelopmental outcomes in low risk, healthy 2-year-olds. This study was a secondary analysis of a nested case-control subgroup from the prospective, maternal-infant Cork Babies after Screening for Pregnancy Endpoints: Evaluating the Longitudinal Impact using Neurological and Nutritional Endpoints (BASELINE) Birth Cohort Study. At 2 years, serum ferritin, Hb and mean corpuscular volume (MCV) were measured and neurodevelopment was assessed using the Bayley Scales of Infant and Toddler Development ( $n$ 87). Five children had Fe deficiency (ferritin $<12 \mu \mathrm{g} / \mathrm{l}$ ) and no child had Fe deficiency anaemia $(\mathrm{Hb}<110 \mathrm{~g} / \mathrm{l}+$ ferritin $<12 \mu \mathrm{g} / \mathrm{l})$. Children with microcytosis (MCV $<74 \mathrm{fl}$, $n$ 13) had significantly lower mean cognitive composite scores (88.5 (sD 13.3) $v \cdot 97 \cdot 0$ (sD 7.8), $P=0 \cdot 04$, Cohen's $d$ effect size $=0 \cdot 8$ ) than those without microcytosis. The ferritin concentration which best predicted microcytosis was calculated as $18.4 \mu \mathrm{g} / \mathrm{l}(\mathrm{AUC}=0.87(95 \% \mathrm{CI}$ $0.75,0.98), P<0.0001$, sensitivity $92 \%$, specificity $75 \%$ ). Using $18.5 \mu \mathrm{g} / 1$ as a threshold, children with concentrations $<18.5 \mu \mathrm{g} / 1$ had significantly lower mean cognitive composite scores (92.3 (sD 10.5) $v .97 \cdot 8$ (sD 8.1), $P=0.012$, Cohen's $d$ effect size $=0 \cdot 6$ ) compared with those with ferritin $\geq 18.5 \mu \mathrm{g} / \mathrm{l}$. All associations were robust after adjustment for potential confounding factors. Despite a low prevalence of Fe deficiency using current diagnostic criteria in this healthy cohort, microcytosis was associated with lower cognitive outcomes at 2 years. This exploratory study emphasises the need for re-evaluation of the diagnostic criteria for Fe deficiency in young children, with further research in adequately powered studies warranted.

Key words: Bayley Scales of Infant and Toddler Development: Microcytosis: Serum ferritin: Neurodevelopment: Cognition

Infants and young children are at particular risk of Fe deficiency, which can lead to microcytosis and anaemia. Term infants are born with Fe reserves that last for about 6 months; however after this the infant relies on dietary intakes ${ }^{(1)}$. In addition to inadequate dietary Fe intakes, other dietary practices including an increased consumption of unmodified cows' milk and reduced consumption of Fe-fortified products can also result in Fe deficiency in infancy and early childhood ${ }^{(2,3)}$. Globally, it is estimated that $25 \%$ of preschool-age children have Fe deficiency anaemia ${ }^{(4)}$, while in Europe, the prevalence of Fe deficiency ranges from 3 to $48 \%$, with the majority of $\mathrm{Fe}$ deficiency anaemia rates reported as below $5 \%^{(5)}$. However, the lack of consensus and therefore, the wide variability in diagnostic criteria, including both biomarkers and thresholds used for Fe deficiency and Fe deficiency anaemia in infants and young children has challenged the interpretation of Fe status worldwide.

Fe is essential for a number of processes in the developing brain; altered neurotransmitter functioning, brain morphology and reduced myelination have all been observed in Fe deficient rodent models ${ }^{(6,7)}$. Studies in humans have consistently reported that Fe deficiency with and without anaemia in infancy

Abbreviations: BASELINE, Babies after Screening for Pregnancy Endpoints: Evaluating the Longitudinal Impact using Neurological and Nutritional Endpoints; BSID, Bayley Scales of Infant and Toddler Development; IQR, interquartile range; MCV, mean corpuscular volume.

* Corresponding author: Dr D. M. Murray, email d.murray@ucc.ie 
and early childhood is associated with poorer cognitive, motor and behavioural outcomes ${ }^{(8,9)}$, with long-lasting effects into adulthood $^{(10)}$.

Most studies exploring associations between Fe and neurodevelopment have focused on 6-24-month-olds in lowresource settings or in low socioeconomic subgroups; few have explored associations in healthy young children in low risk, high-resource settings. Therefore, the aim of this study was to explore associations between Fe status, haematological indices and neurodevelopmental outcomes at 2 years in an apparently healthy, low risk population recruited through a prospective birth cohort in Ireland.

\section{Methods}

\section{Study design and participants}

This study is a secondary analysis of a nested case-control subgroup of participants that were recruited as part of the Cork Babies after Screening for Pregnancy Endpoints (SCOPE): Evaluating the Longitudinal Impact using Neurological and Nutritional Endpoints (BASELINE) Birth Cohort Study between March 2008 and January 2011. The BASELINE Study was established as a follow-on to the SCOPE Ireland pregnancy cohort study where 1768 low risk, nulliparous women were recruited before 15 weeks' gestation from Cork University Maternity Hospital, as part of an international, multicentre study aimed at investigating early indicators of pregnancy complications $^{(11)}$. At 15 weeks' gestation, research midwives collected information on maternal socioeconomic status, occupation, education, relationship status and a complete medical history. Information on nutritional supplement use, recreational activity, cigarette and alcohol use were recorded for the 3-month period before conception and during the first trimester. Maternal anthropometric and clinical measurements were also collected.

Women in the SCOPE Ireland study provided written informed consent to the BASELINE Study for their infants at 15 weeks' gestation ( $n$ 1537). Infants were followed prospectively from birth, with assessments at day 2 and at 2, 6, 12 and 24 months. Assessments at 5 years of age were completed in December 2016. Detailed information on early life environment, diet, lifestyle, health, growth and development was gathered by interviewer-led questionnaires and clinical assessments performed by trained researchers in accordance with the Declaration of Helsinki, with further information on study design and procedures reported previously ${ }^{(12)}$. Ethical approval was granted by the Clinical Research Ethics Committee of the Cork teaching hospitals (ECM 5(9) 01/07/2008) and the study is registered with the US Institutes of Health Clinical Trials Registry (www.clinicaltrials.gov NCT01498965).

\section{Developmental assessment}

Within the BASELINE Study cohort, a nested case-control subgroup ( $n$ 460) of firstborn children was selected at birth for developmental assessment to examine the effect of fetal growth on developmental outcomes ${ }^{(13)}$. A statistician identified the subgroup using the following criteria to select cases: (1) children born small-for-gestational age, defined as a customised birth weight centile (adjusted for maternal height, booking weight, ethnicity, infant sex and gestational age ${ }^{(14)}$ ) less than the 10th centile ( $n$ 80) and (2) children with a body fat percentage (measured by air-displacement plethysmography) at $2 \mathrm{~d}$ less than the 10th centile for gestation and sex ( $n$ 81). The control group consisted of appropriate-for-gestational age (AGA) infants comprised of a stratified random sample taken from across the customised birth weight centiles above the 10th centile ( $n$ 299).

Participants identified as eligible for the nested subgroup at birth were approached at their 24-month assessment to partake in a detailed developmental assessment. The Bayley Scales of Infant and Toddler Development, 3rd edition (BSID-III) ${ }^{(15)}$ was administered by a research psychologist trained in administration and scoring of the BSID-III who was blinded to the status of the participants. The assessment consists of five scales: cognition, receptive communication, expressive communication, fine motor and gross motor, with receptive and expressive communication combined to form a language composite score and fine and gross motor combined to form a motor composite score. Published normative scores for the composite scales of the BSID-U are a mean of 100 with a SD of 15; developmental delay was defined as a score $<85$.

For the secondary, cross sectional analysis of the nested casecontrol subgroup in this study, we only included the healthy, AGA children from the control group to ensure exclusion of those with fetal growth restriction, due to its confounding impact on both Fe status and neurodevelopment ${ }^{(16,17)}$. We also excluded children with acute infections, chronic disease/illness or born premature ( $<37$ weeks' gestation).

\section{Biological samples}

Venous blood was collected from participants at the 24-month assessment, in only those with parental consent. Whole blood was sent to the Haematology Laboratory of Cork University Hospital for determination of $\mathrm{Hb}$ and mean corpuscular volume (MCV) on the Sysmex XE 2100 Automated Hematology System (Sysmex America Inc.). Serum ferritin and high sensitivity C-reactive protein (CRP) were analysed in the laboratory of the Cork Centre for Vitamin D and Nutrition Research, University College Cork, by immunoturbidimetric assay on the RX Monaco Clinical Chemistry Analyser (Randox Laboratories Ltd, Co.). Children with potential infections/inflammation as indicated by an elevated CRP (>5 mg/l) were excluded from analyses.

\section{Statistical analysis}

Data were analysed using IBM SPSS ${ }^{\circledR}$ for Windows ${ }^{\mathrm{TM}}$ version 21 (IBM Corp.). The distribution of data was examined by $Q-Q$ plots and histograms and descriptive statistics were generated; medians and interquartile ranges (IQR) were used for not normally distributed data, whereas means and standard deviations were provided for the BSID-III scores due to their normal distribution. Significant differences between groups in categorical variables were evaluated using $\chi^{2}$ tests and independent $t$ tests 
or Mann-Whitney $U$ test for continuous variables. Effect sizes were calculated using Cohen's $d$ effect size calculation. Given the low numbers with Fe deficiency or Fe deficiency anaemia, associations with individual haematological indices, first as continuous variables and then as categorical variables (assigned using commonly used thresholds) were explored. Univariate and multivariate adjusted linear regression models were developed to estimate the effect of each haematological index on BSID-III scores. Factors explored in the univariate analysis included maternal age, education, occupation, income, relationship status, Fe status, smoking status, BMI, mode of delivery and infant sex, gestational age, birth anthropometric measures and early feeding methods. Potential confounding factors that were retained in the final multivariate models were identified if they were significant at the $10 \%(P<0 \cdot 1)$ level in the univariate analysis. The residuals of the final model were normally distributed and were also assessed for linearity, homoscedasticity, independence and for the presence of any outliers; associations were expressed as adjusted estimates and 95\% CI.

In addition, receiver operating characteristic curve analysis was used to estimate the ferritin concentration cut-point to best predict a MCV $<74 \mathrm{fl}$. The AUC, sensitivity and specificity were calculated and the best possible cut-point for ferritin concentrations was defined at the highest Youden Index ((sensitivity + specificity) -1$)$.

\section{Results}

From the 460 children selected in the nested case-control subgroup, 366 had a complete BSID-Ш assessment at 2 years (forty-two had an incomplete assessment and fifty-two were unable to attend the assessment). Only the children born AGA (control group) were included in this secondary analysis ( $n$ 240). The measurement of haematological indices at 2 years was a prerequisite for this study, therefore children with no blood sample provided at 2 years ( $n$ 144) were excluded. Those with infection/inflammation at 2 years $(n 3)$ or born premature ( $n$ 6) were also excluded to provide a final sample size of eighty-seven children (see Fig. 1). The principal characteristics of those included in this study and their comparisons to the rest of the study cohort that attended the 24-month assessment are presented in Table 1.

Median $\mathrm{Hb}, \mathrm{MCV}$ and serum ferritin concentrations at 2 years of those included in this study are outlined in Table 1. Using WHO definitions, Fe deficiency (serum ferritin $<12 \mu \mathrm{g} / \mathrm{l}$ ) was observed in $6 \%(n 5)$ and no child had Fe deficiency anaemia (serum ferritin $<12 \mu \mathrm{g} / \mathrm{l}+\mathrm{Hb}<110 \mathrm{~g} / \mathrm{l}$ ). Five children were anaemic $(\mathrm{Hb}<110 \mathrm{~g} / \mathrm{l})$ and $15 \%(n 13)$ had a MCV $<74 \mathrm{fl}$, a threshold indicative of microcytosis ${ }^{(3,18,19)}$.

The median age of completion for the BSID-II assessment was $27 \cdot 2$ (IQR 26·0, 28.6) months. The distribution of subscale and

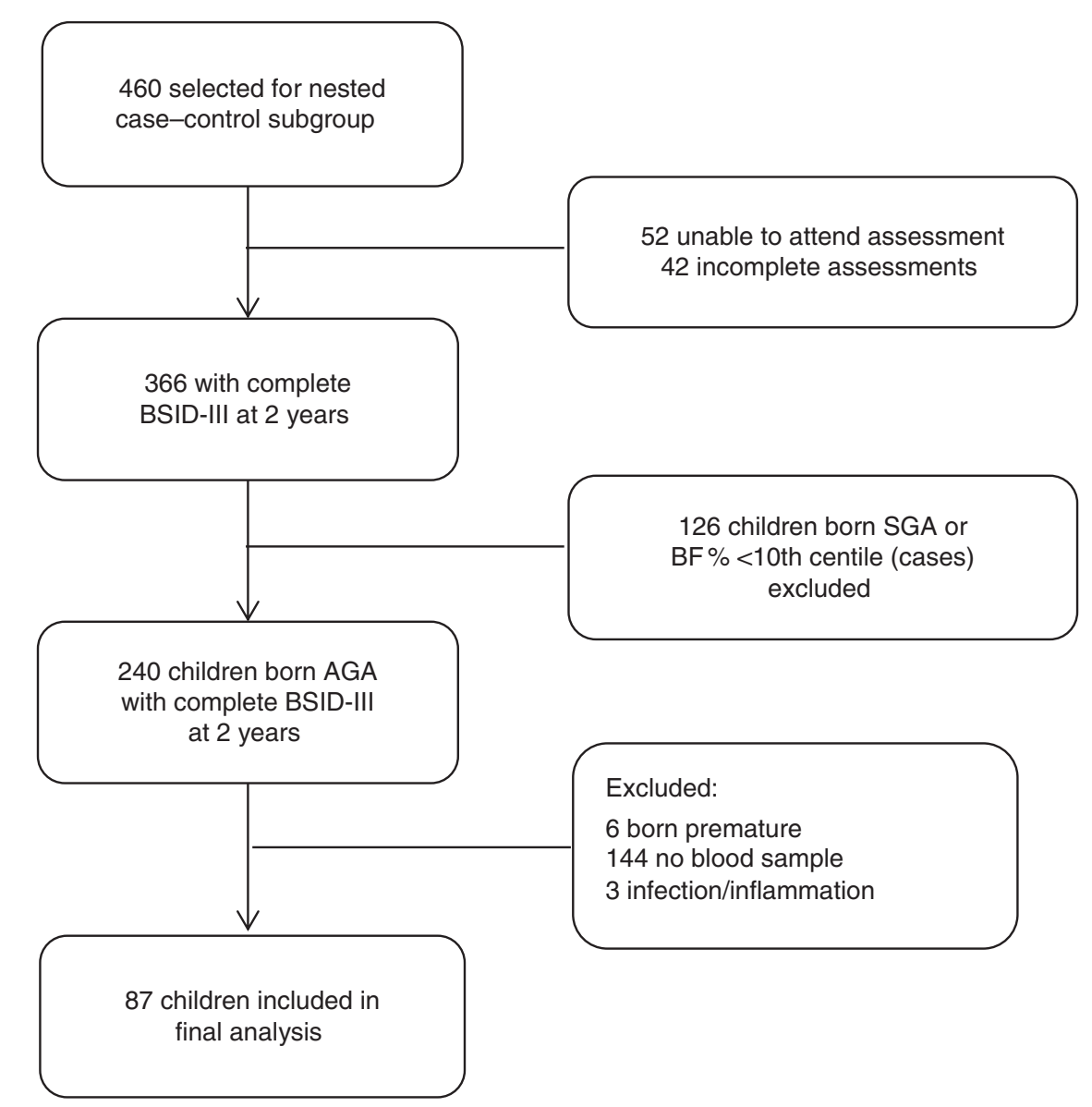

Fig. 1. Flow diagram of study participants. BSID-III, Bayley Scales of Infant and Toddler Development (3rd edition); SGA, small-for-gestational age; AGA, appropriatefor-gestational age; BF, body fat. 
Table 1. Principal characteristics of eligible participants with a blood sample and complete developmental assessment at 2 years $(n 87)$ in comparison to the rest of the participants $(n 1051)$ of the Cork Babies after Screening for Pregnancy Endpoints: Evaluating the Longitudinal Impact using Neurological and Nutritional Endpoints (BASELINE) Birth Cohort Study* (Numbers and percentages; medians and interquartile ranges (IQR))

\begin{tabular}{|c|c|c|c|c|c|}
\hline & \multicolumn{2}{|c|}{ Eligible ( $n$ 87) } & \multicolumn{2}{|c|}{ Not eligible $(n$ 1051) } & \multirow[b]{2}{*}{$P \dagger$} \\
\hline & $n$ & $\%$ & $n$ & $\%$ & \\
\hline \multicolumn{6}{|l|}{ Maternal characteristics } \\
\hline Age (at delivery, years) & & & & & 0.086 \\
\hline Median & \multicolumn{2}{|c|}{31.0} & \multicolumn{2}{|c|}{31.0} & \\
\hline IQR & \multicolumn{2}{|c|}{$28 \cdot 0,33.0$} & \multicolumn{2}{|c|}{$29 \cdot 0,34.0$} & \\
\hline Caucasian & 86 & 99 & 1032 & 99 & 0.999 \\
\hline \multicolumn{5}{|l|}{ Education } & 0.369 \\
\hline$\leq 12$ years & 12 & 14 & 106 & 10 & \\
\hline$>12$ years & 75 & 86 & 942 & 90 & \\
\hline \multicolumn{5}{|l|}{ Marital status } & 0.942 \\
\hline Single & 5 & 6 & 52 & 5 & \\
\hline Married/de facto & 82 & 94 & 999 & 95 & \\
\hline \multicolumn{5}{|l|}{ Household income } & 0.254 \\
\hline$<€ 21000$ & 8 & 9 & 53 & 5 & \\
\hline$€ 21000-€ 63000$ & 38 & 44 & 402 & 40 & \\
\hline$€ 64000-€ 105000$ & 33 & 38 & 414 & 41 & \\
\hline$>€ 105000$ & 8 & 9 & 140 & 14 & \\
\hline \multicolumn{5}{|l|}{ Infant characteristics } & 0.999 \\
\hline Male & 44 & 51 & 542 & 51 & 0.947 \\
\hline \multicolumn{5}{|l|}{ Gestational age (weeks) } & 0.036 \\
\hline Median & \multicolumn{2}{|c|}{$40 \cdot 6$} & \multicolumn{2}{|c|}{$40 \cdot 3$} & \\
\hline IQR & \multicolumn{2}{|c|}{$39 \cdot 6,41 \cdot 3$} & \multicolumn{2}{|c|}{$39 \cdot 3,41 \cdot 0$} & \\
\hline Birth weight $(\mathrm{kg})$ & \multirow{3}{*}{\multicolumn{2}{|c|}{$\begin{array}{c}3.6 \\
3.4,3.8\end{array}$}} & & & 0.010 \\
\hline Median & & & \multicolumn{2}{|c|}{3.5} & \\
\hline IQR & & & \multicolumn{2}{|c|}{$3 \cdot 2,3 \cdot 8$} & \\
\hline Birth length $(\mathrm{cm})$ & & & & 0.022 \\
\hline Median & \multirow{2}{*}{\multicolumn{2}{|c|}{$\begin{array}{c}50 \cdot 8 \\
49 \cdot 8,52 \cdot 0\end{array}$}} & \multirow{2}{*}{\multicolumn{2}{|c|}{$50 \cdot 3$}} & \\
\hline IQR & & & $49 \cdot 0,51 \cdot 8$ & & \\
\hline 24-month assessment: $\mathrm{Hb}(\mathrm{g} / \mathrm{l}) \ddagger$ & \multicolumn{2}{|c|}{$49 \cdot 8,52 \cdot 0$} & & & 0.374 \\
\hline Median & \multirow{2}{*}{\multicolumn{2}{|c|}{$\begin{array}{c}119 \cdot 5 \\
114 \cdot 3,124 \cdot 0\end{array}$}} & \multicolumn{2}{|c|}{$120 \cdot 0$} & \\
\hline IQR & & & 116 & & \\
\hline 24-month assessment: mean corpuscular volume (fl) $\ddagger$ & & & & & 0.557 \\
\hline Median & & & & & \\
\hline IQR & & & & & \\
\hline 24-month assessment: serum ferritin $(\mu \mathrm{g} / \mathrm{l}) \ddagger$ & & & & & 0.552 \\
\hline Median & & & & & \\
\hline IQR & & & & & \\
\hline
\end{tabular}

* Maternal data collected at 15 weeks' gestation unless otherwise stated.

† $P$ values are comparisons between groups with $x^{2}$ or Mann-Whitney $U$ tests.

$\ddagger$ Haematological indices not available in all participants that were not eligible for this study.

composite scores of the BSID-Ш are presented in Table 2. There was a low prevalence of scores indicative of developmental delay; $6 \%(n 5), 6 \%(n 5)$ and $3 \%(n 2)$ had scores $<85$ in the cognition, language and motor composite scales, respectively.

$\mathrm{Hb}$ concentrations at 2 years were not associated with BSID-Ш subscale or composite scores at 2 years. From the univariate regression analysis, MCV was positively associated with BSID-Ш cognitive composite (estimate $0.71 ; 95 \%$ CI 0.03 , $1.40), P=0.041)$ scores, however, this association was no longer significant in the final model adjusted for potential confounding factors (sex, birth weight, maternal education, occupation, household income). Children with microcytosis (MCV < 74fl) had significantly lower BSID-Ш cognitive composite scores than those without microcytosis $(\mathrm{MCV} \geq 74 \mathrm{fl}$ ), with a strong Cohen's $d$ effect size of 0.8 (Table 3). This association remained robust after adjustment for potential confounding factors (adjusted estimate $-6 \cdot 7$; 95\% CI $-12 \cdot 9$,
$-0 \cdot 5, P=0.034$, see online Supplementary Table S1 for full multivariate model). There were no other significant differences in study characteristics between those with and without microcytosis at 2 years.

Of the five participants with cognitive delay (scores $<85$ ), four had microcytosis. Only four children with microcytosis at 2 years had concurrent Fe deficiency, indicated by ferritin concentrations $<12 \mu \mathrm{g} / \mathrm{l}$. When the four children were excluded, those with microcytosis and ferritin concentrations $\geq 12 \mu \mathrm{g} / \mathrm{l}(n$ 9) continued to have lower mean cognitive composite ( 87.2 (SD 15.2) v. 96.9 (sD 7.7), $P=0.069$ ) scores at 2 years, although this association was weaker.

Serum ferritin concentrations were positively associated with BSID-II fine motor subscale scores (estimate 0.04; $95 \% \mathrm{CI}$ $0 \cdot 01,0 \cdot 08, P=0.036)$ at 2 years, which was robust after adjustment for sex, birth weight, maternal education, occupation and household income (adjusted estimate 0.04; 95\% CI 0.01, 0.08, 
Table 2. Distribution of Bayley Scales of Infant and Toddler Development (3rd edition) scores in study participants ( $n$ 87)

(Mean values and standard deviations; medians and interquartile ranges (IQR))

\begin{tabular}{|c|c|c|c|c|}
\hline & Mean & SD & Median & IQR \\
\hline \multicolumn{5}{|l|}{ Subscale scores } \\
\hline Cognition & $9 \cdot 1$ & 1.8 & $9 \cdot 0$ & $8 \cdot 0,10 \cdot 0$ \\
\hline Receptive communication & $10 \cdot 7$ & $2 \cdot 6$ & 11.0 & $9 \cdot 0,13 \cdot 0$ \\
\hline Expressive communication & $11 \cdot 1$ & $3 \cdot 2$ & $12 \cdot 0$ & $9.0,13.0$ \\
\hline Fine motor & 11.5 & $2 \cdot 6$ & 11.0 & $10 \cdot 0,13 \cdot 0$ \\
\hline Gross motor & 9.7 & $2 \cdot 1$ & $10 \cdot 0$ & $8 \cdot 0,11 \cdot 0$ \\
\hline \multicolumn{5}{|l|}{ Composite scores } \\
\hline Cognition & 95.5 & $9 \cdot 2$ & $95 \cdot 0$ & $90 \cdot 0,100 \cdot 0$ \\
\hline Language & $105 \cdot 7$ & $15 \cdot 6$ & $109 \cdot 0$ & $96 \cdot 3,115 \cdot 0$ \\
\hline Motor & $103 \cdot 8$ & 11.4 & $103 \cdot 0$ & $97 \cdot 0,112 \cdot 0$ \\
\hline
\end{tabular}

Table 3. Differences in Bayley Scales of Infant and Toddler Development (3rd edition) subscale and composite scores between those with microcytosis (mean corpuscular volume $(\mathrm{MCV})<74 \mathrm{fl}$ ) and without microcytosis $(\mathrm{MCV} \geq 74 \mathrm{fl})$ at 2 years

(Mean values and standard deviations)

\begin{tabular}{|c|c|c|c|c|c|c|}
\hline & \multicolumn{2}{|c|}{$\begin{array}{c}\mathrm{MCV}<74 \mathrm{fl} \\
\quad(n 13)\end{array}$} & \multicolumn{2}{|c|}{$\begin{array}{l}\mathrm{MCV} \geq 74 \mathrm{fl} \\
\quad(n 55)\end{array}$} & \multirow[b]{2}{*}{$P^{*}$} & \multirow[b]{2}{*}{$P \dagger$} \\
\hline & Mean & SD & Mean & SD & & \\
\hline \multicolumn{7}{|l|}{ Subscale scores } \\
\hline Cognition & $7 \cdot 7$ & $2 \cdot 7$ & 9.4 & 1.6 & 0.044 & $0.036 \ddagger$ \\
\hline $\begin{array}{l}\text { Receptive } \\
\text { communication }\end{array}$ & $9 \cdot 1$ & 3.6 & $10 \cdot 8$ & $2 \cdot 4$ & 0.076 & 0.373 \\
\hline $\begin{array}{l}\text { Expressive } \\
\text { communication }\end{array}$ & $10 \cdot 7$ & $4 \cdot 6$ & $11 \cdot 3$ & $2 \cdot 9$ & 0.615 & 0.721 \\
\hline Fine motor & $10 \cdot 2$ & $2 \cdot 6$ & $11 \cdot 7$ & $2 \cdot 4$ & 0.059 & 0.121 \\
\hline Gross motor & $9 \cdot 3$ & 1.6 & $9 \cdot 7$ & $2 \cdot 4$ & 0.647 & 0.665 \\
\hline \multicolumn{7}{|l|}{ Composite scores } \\
\hline Cognition & 88.5 & $13 \cdot 3$ & 97.0 & 7.8 & 0.044 & $0.036 \ddagger$ \\
\hline Language & 99.9 & 23.4 & $106 \cdot 2$ & 14.4 & 0.291 & 0.390 \\
\hline Motor & 98.6 & 11.6 & 104.5 & 10.9 & $0 \cdot 108$ & 0.173 \\
\hline
\end{tabular}

* $P$ values are comparisons between groups using independent $t$ tests.

$\dagger P$ values from linear regression models adjusted for sex, birth weight, maternal education, occupation and household income.

$\ddagger$ Cohen's $d$ effect size $=0.8$

$P=0.037)$. There were no significant associations between Fe deficiency or Fe deficiency anaemia and BSID-Ш scores at 2 years.

There was a strong positive association between MCV and ferritin concentrations at 2 years (Spearman's $r 0.52, P<0.0001$ ). Children with microcytosis had significantly lower median ferritin concentrations compared with those without microcytosis (13.8 (IQR 11.6, 15.8) v. 21.1 (IQR 18·1, 39.3) $\mu \mathrm{g} / \mathrm{l}, P<0 \cdot 0001$ ). Following this observation, receiver operating characteristic curve analysis was performed to estimate the ferritin concentration to best predict microcytosis in this subgroup of healthy children. The ferritin concentration which best predicted microcytosis was calculated to be $18.4 \mu \mathrm{g} / \mathrm{l}(\mathrm{AUC}=0.87$ (95\% CI $0.75,0.98), P<0.0001$, sensitivity $92 \%$, specificity $75 \%$ ). From this, a threshold of $<18.5 \mu \mathrm{g} / \mathrm{l}$ was subsequently used to investigate associations with BSID-Ш scores. Children with ferritin concentrations $<18.5 \mu \mathrm{g} / \mathrm{l}$ ( $n$ 28) had significantly lower mean BSID-W cognitive composite (92.3 (SD 10.5) v.97.8 (sD 8.1),$P=0 \cdot 012$, Cohen's $d$ effect size $=0 \cdot 6$ ) scores than those with concentrations $\geq 18.5 \mu \mathrm{g} / \mathrm{l}$. After adjustment for confounding factors, ferritin concentrations $<18.5 \mu \mathrm{g} / \mathrm{l}$ remained inversely associated with cognitive composite (adjusted estimate $-6 \cdot 0(95 \% \mathrm{CI}-10 \cdot 2,-1 \cdot 8), P=0 \cdot 006)$ scores at 2 years.

\section{Discussion}

This secondary analysis of a nested case-control subgroup of children from a low risk maternal-infant birth cohort has provided a much-needed exploration of associations between haematological indices and neurodevelopmental outcomes in a high-resource setting. In this apparently healthy cohort, microcytosis, indicated by a MCV $<74 \mathrm{fl}$ was associated with lower cognitive outcomes in 2-year-old children. We also observed lower cognitive scores in children with ferritin concentrations $<18.5 \mu \mathrm{g} / \mathrm{l}$, a threshold we estimated to predict microcytosis in this group of children, highlighting an association between currently defined normal or borderline low ferritin concentrations, as opposed to overt Fe deficiency, and adverse developmental outcomes in children in a highresource setting.

Neither Fe deficiency nor Fe deficiency anaemia was associated with BSID-Ш scores at 2 years in this study, in contrast to findings from earlier studies in more deprived populations ${ }^{(9,20)}$. However, our study was not adequately powered to accurately explore these associations given the low prevalence of $\mathrm{Fe}$ deficiency and Fe deficiency anaemia in our healthy cohort, which we previously attributed to a dietary pattern of increased Fe-fortified product consumption and low intakes of unmodified cows' milk ${ }^{(2)}$. Our rates of Fe deficiency and Fe deficiency anaemia are much lower than contemporary data reported in many other European countries ${ }^{(5)}$ and the USA ${ }^{(21)}$ and are in particular contrast to the last report in Ireland from the 1990's, where $9 \%$ of 2 -year-olds had Fe deficiency anaemia ${ }^{(22)}$. In addition, the cohort assessed in the current study differs from most observational studies in that the majority of previous studies have examined cohorts based in either low-resource settings or in subgroups of low socioeconomic status ${ }^{(20,23)}$. Only two studies in Iceland ${ }^{(24,25)}$ and an earlier study from the $\mathrm{UK}^{(26)}$ have investigated associations in young children in highresource settings similar to the current study. In low-resource settings, the presence of confounding factors which may also impact neurodevelopment including nutritional deprivation and malnourishment can make it difficult to identify Fe deficiency as the sole cause of neurodevelopmental alterations in such populations.

Interestingly, microcytosis indicated by a low MCV at 2 years was associated with significantly lower BSID-Ш cognitive scores, even following adjustment for socioeconomic factors. Linear associations between MCV and behaviour and language/ expression have been observed previously ${ }^{(24,27,28)}$, however, to our knowledge, this is the first study to show an adverse effect of microcytosis on cognition. Microcytosis is characterised by erythrocytes that are smaller than normal. In healthy populations, this presentation is often due to a decreased availability and supply of Fe resulting in a decreased production of $\mathrm{Hb}$, although other causes of microcytosis include anaemia of chronic disease, congenital thalassaemias and sideroblastic 
anaemias $^{(29)}$. However, the microcytosis observed in this study is almost certainly Fe deficiency related. Our cohort was a healthy birth cohort population, with normal CRP levels making chronic inflammation or disease unlikely. Our ethnic diversity is low, $99 \%$ of our cohort were white Caucasian, with no children of African or Mediterranean decent, making thalassaemia an unlikely cause. Although we cannot fully rule out lead poisoning as a cause of acquired sideroblastic anaemia, no sideroblasts were reported on the manual blood films which accompanied our full blood count analysis.

The finding that microcytosis was associated with reduced cognitive outcomes in this low risk population is an important one. It suggests that a low MCV may be a more specific marker of functional Fe deficiency than very low serum ferritin concentrations, which are susceptible to much variation ${ }^{(30)}$. Over two-thirds of those with microcytosis presented with normal serum ferritin concentrations $(\geq 12 \mu \mathrm{g} / \mathrm{l})$, therefore the use of ferritin alone as a diagnostic tool would have resulted in a number of children at risk of poorer cognitive outcomes being undetected. Furthermore, despite the small numbers with cognitive delay in this cohort, it is striking to note that the majority of those with delay had microcytosis. This finding emphasises the need for further consideration of the diagnostic criteria for Fe deficiency in young children, with a need to assess Fe status using multiple haematological indices to ensure no children at risk of physiological or developmental consequences are inadvertently undiagnosed.

In this exploratory analysis, we calculated that a ferritin concentration threshold of $18.4 \mu \mathrm{g} / \mathrm{l}$ was predictive of microcytosis in this cohort, albeit in a small group of children. We observed that children with ferritin concentrations below $18.5 \mu \mathrm{g} / \mathrm{l}$ had significantly lower BSID-Ш cognitive scores at 2 years, raising the possibility that the current commonly used WHO serum ferritin cut-off for Fe deficiency of $12 \mu \mathrm{g} / \mathrm{l}$ may need re-evaluation. Based on our preliminary findings, $18.5 \mu \mathrm{g} / \mathrm{l}$ may be considered a candidate threshold to identify those at risk of adverse clinical outcomes or suboptimal development, especially in high-resource settings, although, further research with adequately powered validation studies are urgently required to ascertain this. This is the first study to show that in a healthy population where very low ferritin concentrations (Fe deficiency) were uncommon, there was still a significant adverse consequence of being in the lower end of the currently defined normal range for ferritin concentrations. Longitudinal studies have previously shown a persistent effect of Fe deficiency at this stage ${ }^{(10)}$, however, the long-term consequences of this borderline low $\mathrm{Fe}$ status at this crucial phase of brain development is yet to be elucidated.

In addition, ferritin concentrations at 2 years were positively associated with BSID-Ш fine motor scores, in accordance with previous studies ${ }^{(25,31)}$. A number of animal studies have suggested altered oligodendrocyte function due to Fe deficiency, resulting in impaired myelination of neurons, affecting motor function. Alterations in monoaminergic pathways, in particular in dopamine metabolism have also been suggested to impact motor development in early childhood ${ }^{(32)}$.

The obligate reliance on global developmental assessments at this age is a limitation in this area of research. Global developmental assessments, including the BSID-Ш, have been criticised for being too broad and not sensitive enough to identify specific cognitive processes that are affected by nutritional factors and do not correlate highly with later tests of intelligence in school-age children ${ }^{(33,34)}$. More specific measures of cognitive tasks, such as tests of memory and learning at school-age may be of more importance to investigating the more subtle effects of Fe deficiency and borderline low Fe status $^{(33)}$.

A potential limitation of this study is that the analysis is crosssectional in design, although it is important to note that prospectively collected maternal and early life data were used in the analysis, thereby enabling deeper interpretation of the data than would be possible with a cross-sectional survey. The prospective design of the Cork BASELINE Birth Cohort Study, with its multidisciplinary team, with both validated nutritional and neurodevelopmental assessments performed by experts in their field are strengths of this study. Our secondary analysis of the nested subgroup within the cohort is limited by the small sample size included, although the eighty-seven children included were representative of the entire BASELINE cohort (see Table 1) and this sample size is comparable to previous observational studies in this field ${ }^{(24,25)}$. However, adequately powered studies are required to further investigate and expand on our findings in this study with the aim to identify the best measures of Fe status in young children. The exploration of associations between Fe deficiency/Fe deficiency anaemia and developmental outcomes were somewhat limited by the small number of children with either condition, however this is reflective of the high-resource population studied and generalisable to other similar low risk, healthy populations. Despite the overall normal developmental profile of the group, we still found a significant functional effect of microcytosis and borderline normal/low Fe status.

Although the public health threat associated with Fe deficiency has diminished in many low risk, high-resource settings, the current study highlights that strategies aimed at preventing Fe deficiency, but also low or suboptimal Fe status in young children in such settings are still pertinent. Despite a low prevalence of Fe deficiency using current diagnostic criteria in this apparently healthy cohort, microcytosis was still associated with lower cognitive outcomes at 2 years. This study further emphasises the need for re-consideration and evaluation of the diagnostic criteria currently used to diagnose Fe deficiency and Fe deficiency anaemia in infants and young children. Further research is warranted to identify the most suitable biomarkers and thresholds to ensure no children at risk of adverse clinical or developmental consequences are inadvertently undiagnosed and untreated.

\section{Acknowledgements}

The authors thank the participants and their families for their continued support and both the SCOPE Ireland and Cork BASELINE Birth Cohort Study research teams.

A grant from the National Children's Research Centre to D. M. M., J. O'B. H., A. D. I., L. C. K. and M. E. K. is the primary funding source for the Cork BASELINE Birth Cohort Study. Additional 
support came from grants from the UK Food Standards Agency to J. O'B. H. and A. D. I. and from Danone Nutricia Early Life Nutrition to M. E. K. and D. M. M. The SCOPE Ireland Study was funded by a grant from the Health Research Board of Ireland (CSA 02/2007) to L. C. K. The INFANT Research Centre is Science Foundation Ireland funded (grant no. 12/RC/2272). No funding agencies had any role in the design, analysis or writing of this article.

E. K. M. carried out data collection, database construction and data analysis and drafted the first and subsequent drafts of the manuscript. D. M. M. is the overall Principal Investigator (PI) of the Cork BASELINE Birth Cohort Study and planned the study design, aided in data analysis and manuscript preparation. M. E. K. is Co-PI and specialist nutrition lead of the Cork BASELINE Birth Cohort Study and aided in study governance, design and manuscript preparation. G. H. and C. A. carried out data collection and database construction. L. C. K., J. O'B. H. and A. D. I. are co-PI of the Cork BASELINE Birth Cohort Study and aided in study governance and edited the final manuscript. L. C. K. is the PI of the SCOPE Ireland pregnancy study. All authors reviewed and approved the final submission.

The authors declare that there are no conflicts of interest.

\section{Supplementary material}

For supplementary material/s referred to in this article, please visit https://doi.org/10.1017/S0007114517001945

\section{References}

1. Agostoni C, Decsi T, Fewtrell M, et al. (2008) Complementary feeding: a commentary by the ESPGHAN Committee on Nutrition. J Pediatr Gastroenterol Nutr 46, 99-110.

2. McCarthy EK, Ní Chaoimh C, Hourihane JO, et al. (2017) Iron intakes and status of 2-year-old children in the Cork BASELINE Birth Cohort Study. Matern Child Nutr 13, e12320.

3. Gunnarsson BS, Thorsdottir I \& Palsson G (2004) Iron status in 2 -year-old Icelandic children and associations with dietary intake and growth. Eur J Clin Nutr 58, 901-906.

4. McLean E, Cogswell M, Egli I, et al. (2009) Worldwide prevalence of anaemia, WHO Vitamin and Mineral Nutrition Information System, 1993-2005. Public Health Nutr 12, 444-454.

5. Eussen S, Alles M, Uijterschout L, et al. (2015) Iron intake and status of children aged 6-36 months in Europe: a systematic review. Ann Nutr Metab 66, 80-92.

6. Lozoff B \& Georgieff MK (2006) Iron deficiency and brain development. Semin Pediatr Neurol 13, 158-165.

7. Lozoff B (2007) Iron deficiency and child development. Food Nutr Bull 28, 4 Suppl., S560-S571.

8. Lozoff B, Beard J, Connor J, et al. (2006) Long-lasting neural and behavioral effects of iron deficiency in infancy. Nutr Rev 64, 5 Pt 2, S34-S43; discussion S72-S91.

9. Grantham-McGregor S \& Ani C (2001) A review of studies on the effect of iron deficiency on cognitive development in children. J Nutr 131, 2S-2, 649S-666S; discussion 666S-668S.

10. Lozoff B, Smith JB, Kaciroti N, et al. (2013) Functional significance of early-life iron deficiency: outcomes at 25 years. J Pediatr 163, 1260-1266.

11. Kenny LC, Black MA, Poston L, et al. (2014) Early pregnancy prediction of preeclampsia in nulliparous women, combining clinical risk and biomarkers: the Screening for Pregnancy
Endpoints (SCOPE) international cohort study. Hypertension 64, 644-652.

12. O'Donovan SM, Murray DM, Hourihane JO, et al. (2015) Cohort profile: The Cork BASELINE Birth Cohort Study: Babies after SCOPE: Evaluating the Longitudinal Impact on Neurological and Nutritional Endpoints. Int J Epidemiol 44, 764-775.

13. O'Neill SM, Hannon G, Khashan AS, et al. (2017) Thinfor-gestational age infants are at increased risk of neurodevelopmental delay at 2 years. Arch Dis Child Fetal Neonatal Ed 102, F197-F202.

14. McCowan LM, Thompson JM, Taylor RS, et al. (2013) Clinical prediction in early pregnancy of infants small for gestational age by customised birthweight centiles: findings from a healthy nulliparous cohort. PLOS ONE 8, e70917.

15. Bayley N (2006) The Bayley Scales of Infant and Toddler Development, 3rd ed. San Antonio, TX: The Psychological Corporation.

16. Savchev S, Sanz-Cortes M, Cruz-Martinez R, et al. (2013) Neurodevelopmental outcome of full-term small-for-gestationalage infants with normal placental function. Ultrasound Obstet Gynecol 42, 201-206.

17. Rao R \& Georgieff MK (2007) Iron in fetal and neonatal nutrition. Semin Fetal Neonatal Med 12, 54-63.

18. Thorisdottir AV, Thorsdottir I \& Palsson GI (2011) Nutrition and iron status of 1-year olds following a Revision in infant dietary recommendations. Anemia 2011, 986303.

19. Vendt N, Grunberg H, Leedo S, et al. (2007) Prevalence and causes of iron deficiency anemias in infants aged 9 to 12 months in Estonia. Medicina (Kaunas) 43, 947-952.

20. Lozoff B, Brittenham GM, Wolf AW, et al. (1987) Iron deficiency anemia and iron therapy effects on infant developmental test performance. Pediatrics 79, 981-995.

21. Brotanek JM, Gosz J, Weitzman M, et al. (2007) Iron deficiency in early childhood in the United States: risk factors and racial/ ethnic disparities. Pediatrics 120, 568-575.

22. Freeman VE, Mulder J, van't Hof MA, et al. (1998) A longitudinal study of iron status in children at 12,24 and 36 months. Public Health Nutr 1, 93-100.

23. Shafir T, Angulo-Barroso R, Jing Y, et al. (2008) Iron deficiency and infant motor development. Early Hum Dev $\mathbf{8 4}$, 479-485.

24. Gunnarsson BS, Thorsdottir I, Palsson G, et al. (2007) Iron status at 1 and 6 years versus developmental scores at 6 years in a well-nourished affluent population. Acta Paediatr 96, 391-395.

25. Thorisdottir AV, Gunnarsdottir I, Palsson GI, et al. (2013) Iron status and developmental scores in 6-year-olds highlights ongoing need to tackle iron deficiency in infants. Acta Paediatr 102, 914-919.

26. Sherriff A, Emond A, Bell JC, et al. (2001) Should infants be screened for anaemia? A prospective study investigating the relation between haemoglobin at 8,12 , and 18 months and development at 18 months. Arch Dis Child 84, 480-485.

27. Johnson SR, Winkleby MA, Boyce WT, et al. (1992) The association between hemoglobin and behavior problems in a sample of low-income Hispanic preschool children. J Dev Behav Pediatr 13, 209-214.

28. Ruff HA, Markowitz ME, Bijur PE, et al. (1996) Relationships among blood lead levels, iron deficiency, and cognitive development in two-year-old children. Environ Health Perspect 104, 180-185.

29. DeLoughery TG (2014) Microcytic anemia. N Engl J Med 371, 1324-1331.

30. Domellof M, Braegger C, Campoy C, et al. (2014) Iron requirements of infants and toddlers. J Pediatr Gastroenterol Nutr 58, 119-129. 
31. Shafir T, Angulo-Barroso R, Calatroni A, et al. (2006) Effects of iron deficiency in infancy on patterns of motor development over time. Hum Mov Sci 25, 821-838.

32. Doom JR \& Georgieff MK (2014) Striking while the iron is hot: understanding the biological and neurodevelopmental effects of iron deficiency to optimize intervention in early childhood. Curr Pediatr Rep 2, 291-298.
33. Wainwright PE \& Colombo J (2006) Nutrition and the development of cognitive functions: interpretation of behavioral studies in animals and human infants. Am J Clin Nutr 84, 961-970.

34. Canals J, Hernandez-Martinez C, Esparo G, et al. (2011) Neonatal Behavioral Assessment Scale as a predictor of cognitive development and IQ in full-term infants: a 6-year longitudinal study. Acta Paediatr 100, 1331-1337. 